\title{
Active miniature radio frequency field probe
}

\section{A. Glasmachers}

Fachbereich Elektrotechnik und Informationstechnik, Universität Wuppertal, Fuhlrottstrasse 10, 42097 Wuppertal, Germany

\begin{abstract}
For the measuring of the electromagnetic interference (e.g. on men) of RF fields produced by mobile communication equipment field probes are required with high spatial resolution and high sensitivity. Available passive probes show good results with respect to bandwidth and low field distortion, but do not provide the required sensitivity and dynamic range. A significant limitation for active miniature probes is the power supply problem, because batteries cannot be used. Therefore the effect of high impedance connection lines is examined by a numerical field simulation. Different approaches for the design of an active probe are discussed, a favourable solution with a logarithmic demodulator is implemented and measuring results are presented.
\end{abstract}

\section{Introduction}

Radio frequency field probes were originally designed for measurements of antenna systems, since some decades additional applications arise in the field of electromagnetic compatibility. New requirements were caused by the introduction of mobile communication. Radio frequency field probes described in this paper are used to examine health hazards among other measurements. These measurements require following points:

- measuring of the electrical field

- frequency range $800 \mathrm{MHz}-2,4 \mathrm{GHz}$

- spatial resolution $10 \mathrm{~mm}$

- high sensitivity

- high dynamic range

- compatible with new modulation techniques (e.g., CDMA)

Correspondence to: J. Winter

(winter@wemess2.elektro.uni-wuppertal.de)
Because of the required spatial resolution the admissible size of the dipole antenna is limited to $2 \mathrm{~h}<10 \mathrm{~mm}$. Therefore for the power supply of the probe a battery which is much larger then the antenna cannot be used because it will produce inadmissible field distortions. For an external power supply and for the measuring signal transmission two solutions are applicable:

a) An optical method (which is very difficult to implement for miniature probes) or

b) a connection via high impedance lines which will not significantly influence the RF field.

Most of the passive probes and the active probe presented in this paper use the connection via high impedance lines.

\section{Principles and limits of passive field probes}

Passive RF filed probes, which fulfill the frequency range and the spatial resolution requirements, are commercially available (Fig. 1).

The design is based on a long insulating carrier material with the antenna and a detection unit at the measuring end and a box with battery and post processing electronics at the other end. For the detection of the RF signal either a rectification by a Schottky diode or the conversion into heat by a resistor are used (Fig. 2). With respect to sensitivity the rectification gives the better result.

For a short dipole antenna with the length $2 \mathrm{~h}$ placed in parallel to the electrical field $E$ the open circuit voltage $v_{0}$ is given by

$v_{0} \approx 2 \mathrm{~h} E$

and the source impedance by

$Z_{0} \approx-j 37 \Omega \frac{\lambda}{2 \mathrm{~h}}$

For a frequency of $f=1 \mathrm{GHz}(\lambda=300 \mathrm{~mm})$ and an antenna length of $2 \mathrm{~h}=10 \mathrm{~mm}$ this impedance of $Z \approx-j 1.1 \mathrm{k} \Omega$ represents a capacitance of $C_{0} \approx 0.14 \mathrm{pF}$. 


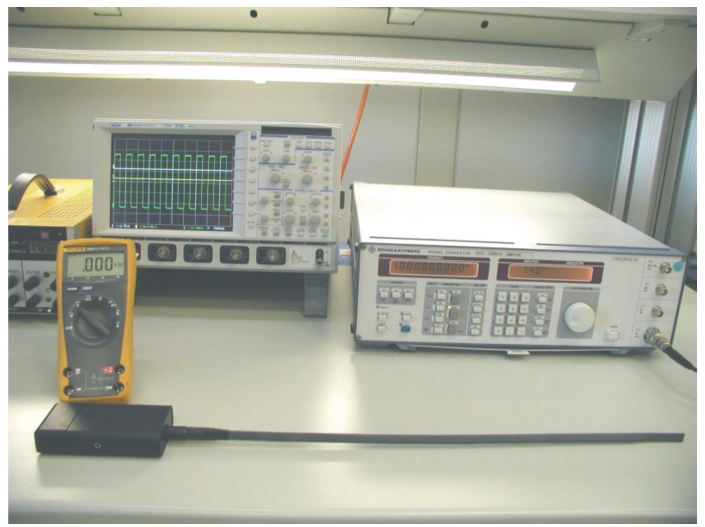

Fig. 1. Commercially available passive field probe.

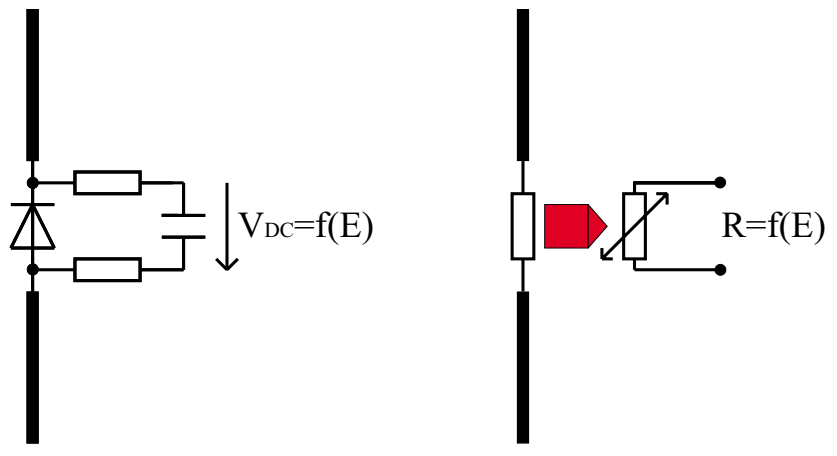

Fig. 2. Principles of passive field probes.

For the Schottky diode the relation of diode current $I_{D}$ und diode voltage $V_{D}$ is given by

$I_{D}=I_{S}\left\{\exp \left(V_{D} / V_{T}\right)-1\right\}$

with $I_{S}$ : saturation current,

$V_{T}:$ temperature voltage

which for small voltages $V_{D}$ can be approximated by

$I_{D} \approx I_{S}\left\{V_{D} / V_{T}+1 / 2\left(V_{D} / V_{T}\right)^{2}\right\}$

The detection effect at low levels is given by the quadratic term in Eq. (4), therefore an approximately quadratic interrelationship of field strength and rectifier voltage with low sensitivity (useful only for field strength $E>10 \mathrm{~V} / \mathrm{m}$ ) and low dynamic range (less than one decade) is achieved.

Another problem of a passive field probe is the high cross sensitivity against low frequency electrostatic noise signals. The large noise signal given in Fig. 4 was produced when the shoe of the operator slipped over the floor covering.

\section{Power supply of an active electric field probe}

The power supply of an active electric field probe by optical transmission is a significant problem for miniature probes. Batteries cannot be used because of their large dimensions which will disturb the rf field. A better solution is to supply

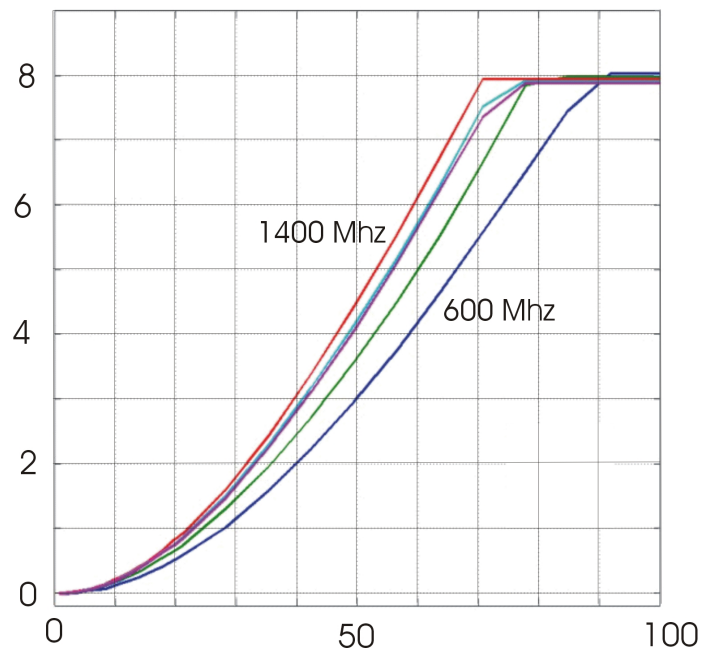

Fig. 3. Properties of a passive field probes with a Schottky diode, $x$-axis: field strength $E[\mathrm{~V} / \mathrm{m}], y$-axis: dc output voltage [V].

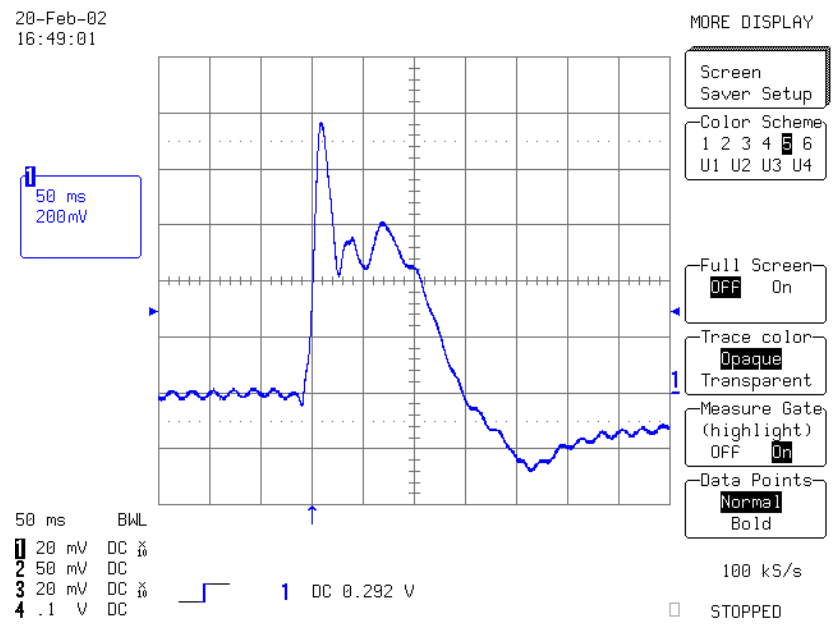

Fig. 4. Effect of low frequency electrostatic noise signals.

power over high resistance lead wires. A MAFIA simulation (Fig. 5) shows that a positive result is achieved with a lead wire resistance of $R L^{\prime}>1 \mathrm{k} \Omega / \mathrm{cm}$. A lead wire length of approximately $50 \mathrm{~cm}$ is shown in Fig. 6 with a resistance of $50 \mathrm{k} \Omega$ of the supply and signal lead. The electrical supply current Isupply must not exceed a few $\mathrm{mA}$ to make sure that the supply voltage stays in a range with no need of special safety measures (e.g. $<500 \mathrm{~V})$.

\section{Active circuits for electric field probes}

The market volume of electric field probes is very low. For this financial reason it is not possible to design a special integrated circuit. Integrated circuits for measuring the transmit signal in mobile radio devices can be used for electric field probes. There are 4 classes of circuits available:

a) RF preamplifier in front of a passive rectifier 


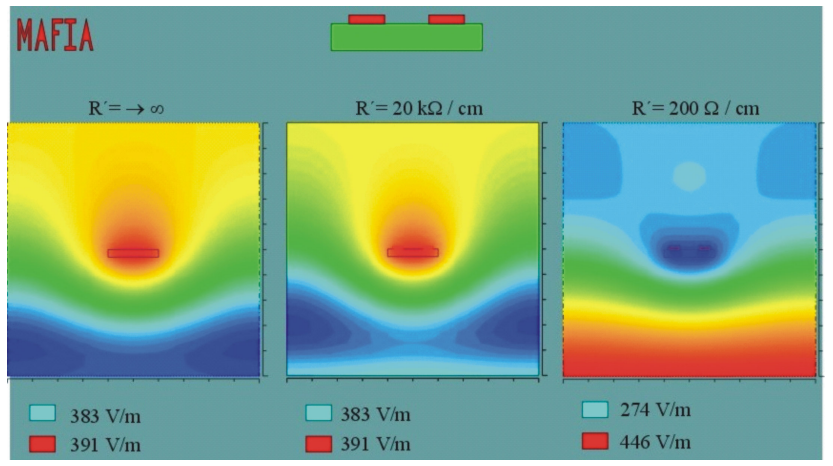

Fig. 5. Field simulation for different lead wire resistances.

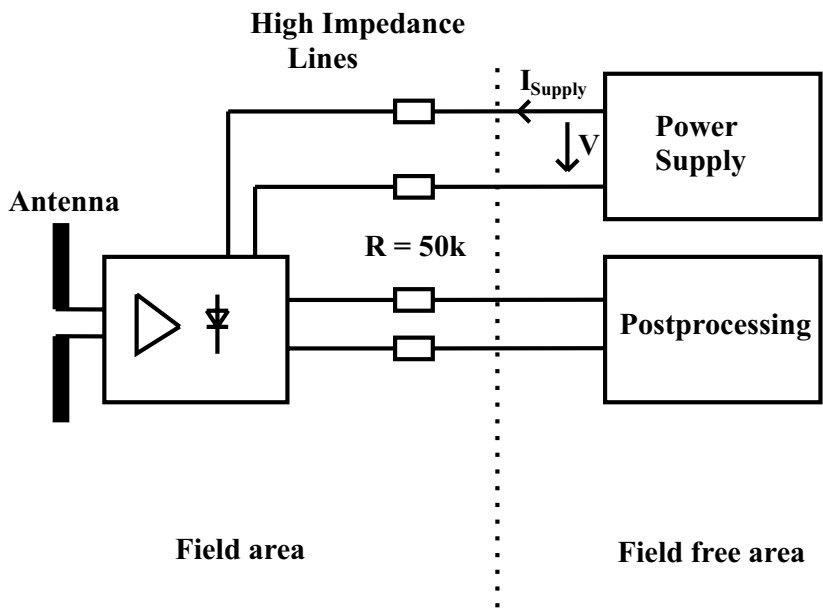

Fig. 6. Power supply of the electric field probe.

b) rectifier with Schottky diodes with bias current and DC buffer amplifier (e.g. LTC5505, Linear Technology)

c) RMS rectifier (e.g. AD8361, Analog Devices)

d) logarithmic demodulator (e.g. AD8314/MAX4000, Analog Devices / Maxim)

a) RF preamplifier

An increase of sensitivity can be achieved e.g. by use of an RF amplifier in front of the Schottky rectifier. RF amplifiers are available in small packages, but there is a need for extra circuitry and for a very high supply current of $I_{\text {supply }}>15 \mathrm{~mA}$.

b) Rectifier with Schottky diodes with bias current and DC buffer amplifier

At the input of these integrated circuits a Schottky diode is used as rectifier. The efficiency is improved by a DC no-load current (bias current). The additional circuitry produces a higher input capacitance which reduces the signal level (capacitive voltage divider). Therefore for this application there is no advantage in sensitivity compared to the passive circuitry. The internal dynamic compression improves the usable dynamic range significantly.

c) RMS rectifier

RMS rectifiers (e.g. AD8361) do not measure the peak of the HF field like the other methods but the root mean square value. RMS rectifiers qualify to measure complex signals (e.g. Code Division Multiple Access used with UMTS). Currently those components have a low power consumption ( $I_{\text {supply }}<2 \mathrm{~mA}$ ) but a small dynamic range of $25 \mathrm{~dB}$ and a low sensitivity. A newer version (AD8362) has a much higher dynamic range of $60 \mathrm{~dB}$, but a very high supply current of $I_{\text {supply }}>20 \mathrm{~mA}$ which is not acceptable for electric field probes.

d) Logarithmic demodulator

A favorable alternative are logarithmic demodulators on a chip. The block diagram of the chip AD8314 is shown in Fig. 7. The supply current is approximately $4 \mathrm{~mA}$. This circuit provides high sensitivity and high dynamic range.

\section{Example for an active electric field probe}

The circuit of a electric field probe with a dipole of $2 \mathrm{~h}=$ $10 \mathrm{~mm}$ length with a logarithmic demodulator AD8314 is shown in Fig. 8 on a printed circuit board made out of FR4 (still without high resistant lead wires). The high resistant lead wires for power supply are placed on one side of the PCB, the high resistant wires for the signals on the opposite side. Graphite spray is used for the lead wires.

The measurement of the active field probe was done in a TEM cell. The measurement results compared to a commercial passive probe with the same antenna length are shown in Fig. 9. The advantages are the logarithmic characteristic, higher sensitivity and the enhanced dynamic range.

\section{Summary and outlook}

Integrated circuits for measuring transmit power in mobile radio devices are particularly suitable for use in active electric field probes. Both applications have several identical requirements as frequency range, small package and low power consumption.

Currently available integrated circuits make it possible to build electric field probes which provide remarkably better sensitivity and dynamic range compared to passive probes. In the near future newer components are expected, respectively announced, to have more improvements in supply current and the ability to measure RMS values of complex signals. 


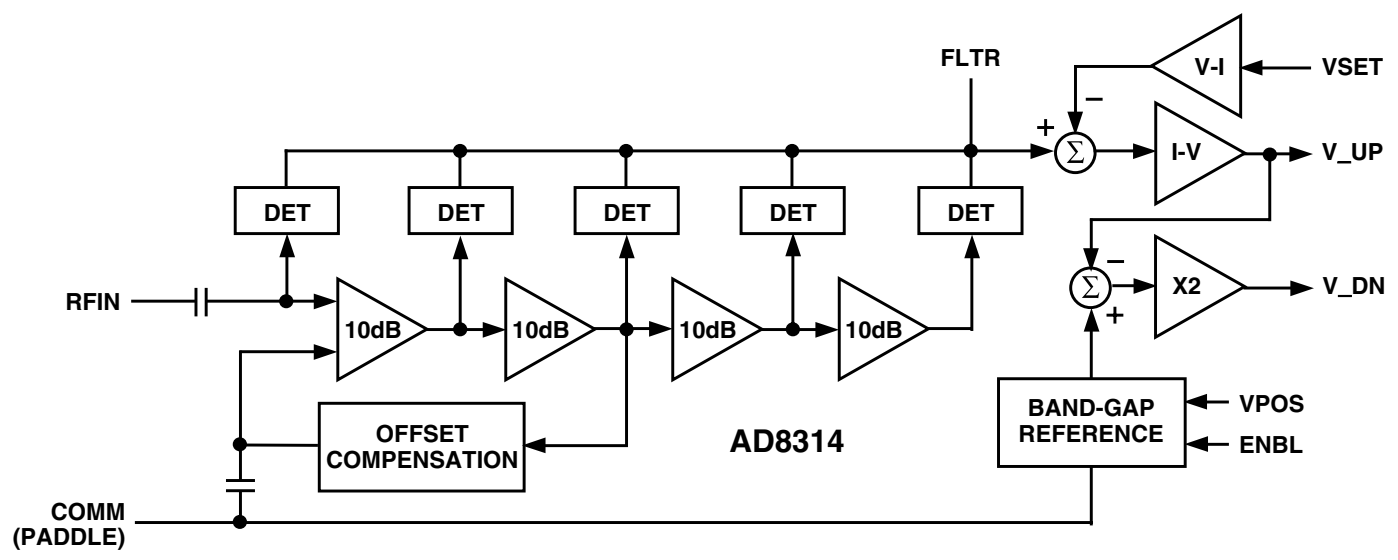

Fig. 7. Block diagram of the logarithmic demodulator AD8314.

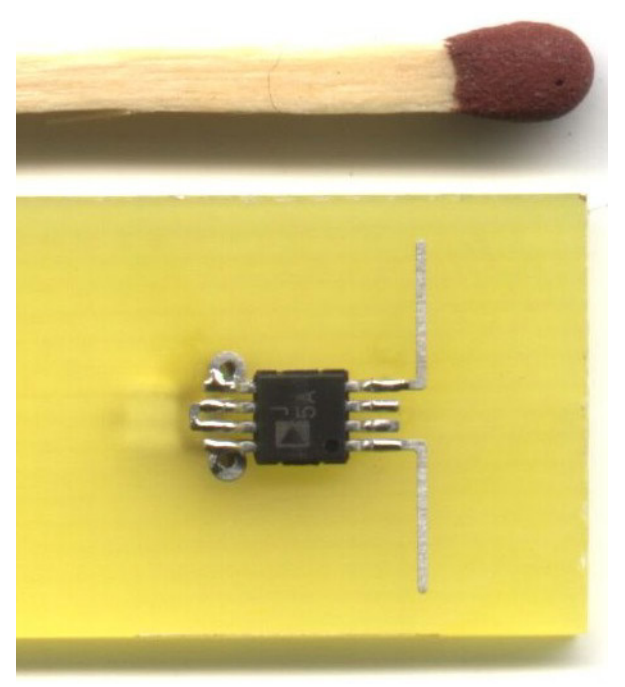

The technique of using high resistance lead wires for power supply has a very small effect on the field to be measured. The technological realization of these wires is still insufficient; a better replacement for the graphite spray to form the high resistant lead wires is needed.

Fig. 8. Layout of an active electric field probe.

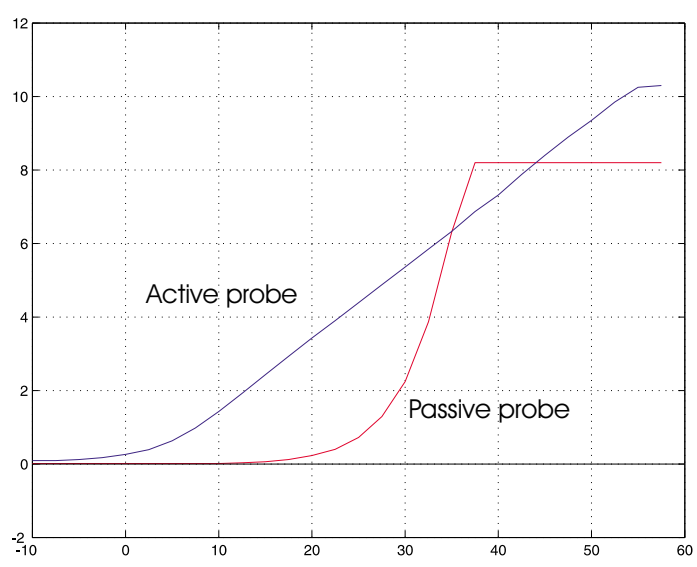

Fig. 9. Characteristics of field probes with $x$-axis: field strength $E$ [dBV/m], $y$-axis: dc output voltage [V]. 